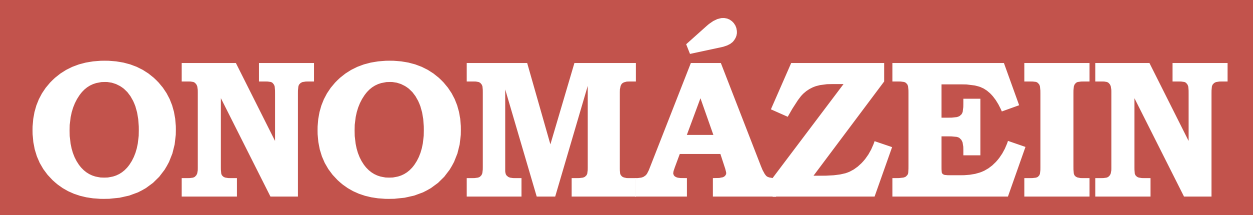

Revista de lingüística, filología y traducción

\title{
N. de la T.: dícese de un elemento controvertido. Análisis de la recepción de las notas del traductor en la traducción del chino de Diarios del Sáhara
}

Taking note of translator's notes. Analysis of the reception of the translator's notes in the Chinese translation of Stories of the Sahara

\section{Sara Rovira-Esteva \\ Universidad Autónoma de Barcelona España}

\section{Irene Tor-Carroggio}

Universidad Autónoma de Barcelona

España

\section{(c) $\underset{\mathrm{BY}}{(} \bigodot_{\mathrm{ND}}$}

Sara Rovira-Esteva: Departamento de Traducción e Interpretación y de Estudios del Asia Oriental, Universidad Autónoma de Barcelona, España. | E-mail: sara.rovira@uab.cat

Irene Tor-Carroggio: Departamento de Traducción e Interpretación y de Estudios del Asia Oriental, Universidad Autónoma de Barcelona, España. | E-mail: irene.tor@uab.cat 


\section{Resumen}

Este trabajo se centra en un aspecto del texto literario que a menudo suscita debate y polémica: las notas del traductor. Sin embargo, este controvertido elemento paratextual no ha despertado demasiado interés dentro de la traductología y apenas existen estudios centrados en su recepción por parte de los lectores. Por ello, el objetivo principal de este trabajo era realizar un análisis cuantitativo del grado de aceptación de las notas mediante un cuestionario tomando la traducción del chino de Diarios del Sáhara como estudio de caso. Los resultados apuntan a que el grado de aceptación de las notas en general por parte de los lectores es bastante alto e incluso las consideran muy necesarias cuando son metalingüísticas o intertextuales. En definitiva, adoptando un enfoque innovador, esta investigación ofrece datos objetivos sobre la adecuación y aceptabilidad de las notas del traductor, lo cual constituye una aportación interesante no solo para la disciplina, sino también para traductores y editores a la hora de tomar decisiones informadas sobre la opinión y las necesidades del lector.

Palabras clave: nota del traductor; paratexto; literatura china; recepción; traducción.

\section{Abstract}

This study focuses on an aspect from literary texts that often generates debate and stirs up controversy: translator's notes. However, this polemic paratextual element has not raised much interest in Translation Studies so far and the studies delving into its reception by readers are scarce. Therefore, the main objective of this study was to carry out a quantitative analysis of the notes' degree of acceptance through a questionnaire taking the translation of the Chinese Stories of the Sahara as a case study. The results suggest that, in general, the degree of acceptance of the notes is rather high and they are even considered very necessary when providing metalinguistic or intertextual information. In short, by having adopted an innovative approach, this research offers objective data on the adequacy and acceptability of translator's notes, which is an interesting contribution not only to the discipline itself, but also for translators and editors when making informed decisions about the opinion and needs of the reader.

Keywords: translator's notes; paratext; Chinese literature; reception study; translation. 


\section{Introducción}

Decía el escritor francés Alain que la nota es aquel elemento mediocre que pende de algo bello (Genette, 1997: 319). La nota, entendida como "advertencia, explicación, comentario o noticia de cualquier clase que en impresos o manuscritos va fuera del texto" según el Diccionario de la Real Academia Española, es uno de los paratextos más controvertidos por, supuestamente y entre otros motivos, poner en jaque - ¿o quizás a prueba? - la atención del lector. Fancher (2002), editor del Journal of the History of the Behavioral Sciences, confesó tener ciertos prejuicios contra ellas, por lo que suele pedir a los autores que las acorten o eliminen, o bien que las incorporen en el cuerpo del texto. En el ámbito de la traducción existen distintas normas, en el sentido propuesto por Toury (2002), según la cultura de llegada y, por ejemplo, en España parece que hay una mayor tolerancia hacia su presencia en las obras de ficción que en países de habla inglesa, donde hay más tendencia a adoptar técnicas de traducción que invisibilizan al traductor (Sierra, 2008: 273). A pesar de ello, todo traductor o aspirante a serlo ha recibido alguna vez en su vida la directriz de evitarlas al máximo. Por ejemplo, en su Manual de traducción inglés-castellano, López Guix y Minett (1997: 290) aconsejan lo siguiente:

En principio, en una obra literaria es preferible no recurrir al aparato crítico de las notas, aunque puede ocurrir que el traductor tenga que decidir entre diluir una alusión de tipo sociocultural que dota al texto de cierto tono local y mantener dicha alusión por medio de una nota.

Descendiente de las glosas, cuya primera aparición en España se remonta a los siglos IX-X de la mano de las Glosas Emilianenses (Gordeew, 2009), la nota ha sido un elemento controvertido desde el punto de vista literario por considerarse que entorpece la lectura e impide que el texto fluya como si hubiera sido escrito originalmente en el idioma de llegada. Llama la atención que Genette en su obra seminal sobre los paratextos no menciona la traducción como paratexto en sí mismo, y menos aún las notas del traductor. Sin embargo, cabe destacar que dentro del ámbito de la traductología las notas del traductor tampoco han despertado demasiado interés entre los investigadores.

Entre los pocos estudios que existen sobre este tema, los hay centrados en ofrecer una clasificación de acuerdo con su tipología, como los trabajos de Peña y Hernández (1994: 37), Marrero-Pulido (2001) y Toledano (2010). Otros Ilevan a cabo un análisis crítico o descriptivo sobre su necesidad o función en alguna obra en concreto. Así, por ejemplo, Henry (2000) se centra en la traducción al francés de Small World, de David Lodge; El-Madkouri Maataoui (2001) analiza el uso y función de las notas en diferentes traducciones del árabe al español; Ribelles (2004) revisa las notas de las versiones al español de las novelas de Patrick de Modiano; Varney (2007) examina el caso de la traducción al italiano de Lady Chatterley's Lover, de D. H. Lawrence; Martin (2008) estudia las notas de Georg Forster en Nachrichten von den Pelew-Inseln; Sierra (2008), por su parte, compara varias traducciones de El mundo de Sofía a diferentes lenguas; Kamal (2011) revisa diferentes traducciones de el Corán al español; y Valverde (2017) analiza las notas en la traducción independiente de la trilogía de las Cincuenta sombras de Grey. 
Dominan, pues, los enfoques cualitativos cuyos resultados no son extrapolables al conjunto de las notas. Excepto el caso de Wu Pei-Chuan (2013), quien en su tesis doctoral analiza la pertinencia de inclusión u omisión de las notas relacionadas con referentes culturales de dos obras españolas traducidas al chino mediante un cuestionario a los lectores, hasta la fecha no nos consta que se haya publicado ningún otro estudio cuantitativo llevado a cabo desde el punto de vista de la recepción de las notas por parte de los lectores de la traducción.

Aunque la nota al pie ha llegado a ser considerada como un síntoma del fracaso del traductor (Marrero-Pulido, 2001: 71), es innegable que se trata de un recurso de ampliación legítimo recogido por Molina y Hurtado (2002: 510), por ejemplo- para explicitar información que se le presupone al lector de la obra original y al que el traductor puede recurrir para trasladar el texto con fidelidad a la lengua meta. No obstante, para López Guix y Minett (1997: 289), "[I]a introducción en el propio texto de informaciones que el traductor considera necesarias suele ser preferible a las notas al pie de página, que tienen el grave inconveniente de interrumpir el ritmo de la lectura, demasiadas veces de modo innecesario". Newmark (1988: 92) las considera molestas si son largas y numerosas. Así pues, y tal como apunta Marrero-Pulido (2001: 70), las notas del traductor deben analizarse desde la perspectiva de la pragmática de la comunicación, ya que ayudan a recuperar toda aquella información subyacente e implícita que el lector no siempre capta. Ahora bien, el traductor debe encontrar el difícil equilibrio entre lo estrictamente necesario y lo claramente prescindible, lo cual depende de múltiples factores.

La distancia cultural y temporal pueden influir en la decisión a la hora de incluir una nota del traductor. Este tiene en mente a un lector prototípico del que espera determinados conocimientos de la cultura original. Sin embargo, por un lado, los lectores son un público heterogéneo que no tiene por qué responder a un único patrón y, por el otro, el conocimiento del Otro va evolucionando y es desigual entre lectores. Además, mientras que unos buscan que el texto les atrape independientemente de su origen, otros se acercan a la literatura ajena precisamente por su valor documental y como vía de conocimiento de su cultura, como puede ser el caso de la literatura china en España (Marín-Lacarta, 2012). Por lo tanto, es posible que los lectores no compartan la misma actitud, gustos o expectativas hacia las notas al pie en función de su perfil. Por todo ello, coincidimos con López Guix y Minett (1997: 290) en que,

[s]i bien las notas pueden facilitar la lectura y, en la medida en que la explicación ha supuesto una labor de búsqueda por parte del traductor, dejan constancia de ese esfuerzo, puede ocurrir que el lector de la traducción —un lector que ya ha sido seleccionado por el propio texto- posea los conocimientos de los que el traductor carecía y perciba como superflua la aclaración. En este caso, lo más usual es que el lector acabe siendo presa de un sentimiento de irritación. Por ello, el recurso a la nota del traductor debe ser fruto de una elección bien meditada.

Pellat (2018: 169) se expresa en la misma línea en relación concretamente con las notas del traductor en las traducciones del chino: 
Footnotes and endnotes are a sensitive area: many readers and writers do not like their intrusive and distracting nature, but they may occasionally offer insights into peripheral factors. In a sense, the presence of footnotes implies that the text falls short in some way. Not everyone needs notes, for the scope of readers' knowledge is varied and unknown to the author and the translator. Readers learn from the text, by inferring, and excessive explicitation on the part of the translator may be inappropriate. Where notes are used in a text to explain a culture-specific item, the note may not need to be translated: a Chinese footnote on a European celebrity or culture-specific item would probably not need to be translated into, for example, English. Similarly, in a work translated into Chinese, notes on China-specific items such as names of Chinese leaders and celebrities may not need a note in the Chinese target text. Conversely, a translator may need to add a note where culture-specific material is not noted or explained in the core text. Unnecessary notes are sometimes used (perhaps unconsciously) both by writers and translators as a means of demonstrating erudition, but this is irksome for the reader, who may feel insulted and patronised.

El tipo de texto también puede ser un factor determinante en la actitud sobre las notas. En este sentido, Newmark (1988: 93) defiende que en determinados géneros el traductor debería incluso evitar su invisibilidad:

If you are translating an important book, you should not hesitate to write a preface and notes to discuss the usage and meanings of the author's terms, particularly where you sacrifice accuracy for economy in the translation, or where there is ambiguity in the text. In the case of a scholarly work, there is no reason why the reader should not be aware of the translator's informed assistance both in the work and the comment. The artistic illusion of your non-existence is unnecessary.

La cuestión de las notas en relación con la agencia y visibilidad del traductor y la teoría de los polisistemas son fundamentales para entender todos los elementos que entran en juego al determinar el método traductor, es decir, la decisión inicial a la hora de emprender un proyecto de traducción que afecta el texto en su conjunto (Molina y Hurtado, 2002: 507), pero dicho análisis queda fuera del alcance del presente estudio por limitaciones de espacio. En todo caso, teniendo en cuenta las reticencias que suscita este elemento paratextual por parte de los editores y el debate que despierta entre traductores y lectores, este trabajo pretende abordar la cuestión de las notas del traductor, sobre todo desde la perspectiva del lector, tomando como estudio de caso la traducción del chino al español y catalán de la obra Diarios del Sáhara (Shahala de Gushi).

Diarios del Sáhara es la obra más conocida de la escritora taiwanesa Chen Ping, o Echo Chen en Occidente, aunque es más conocida como Sanmao en el universo literario, que fue traducida por primera vez en España en el año 2016 por las autoras de la presente investigación. La obra versa sobre las experiencias del matrimonio formado por un español y la propia autora durante su estancia de varios años en el Sáhara español en la década de los setenta. El texto integra múltiples referencias a los lugares a los que está ligada vitalmente la autora en mayor o menor medida, a saber: la España peninsular, el Sáhara español, China y Taiwán. 
Para alcanzar nuestro objetivo, este estudio se propuso responder las siguientes cuatro preguntas de investigación:

a) ¿Hasta qué punto la política editorial del sello responsable de Diarios del Sáhara en cuanto a las notas del traductor incidió sobre el resultado final?

b) ¿Cuál es la actitud de los lectores acerca de las notas del traductor en general?

c) ¿Qué tipo de nota consideran más necesario los lectores en Diarios del Sáhara?

d) ¿Pueden priorizarse las notas según tipología siguiendo criterios objetivos?

Estas preguntas llevaban asociadas tres hipótesis de partida. En primer lugar, a pesar de que la editorial permite las notas del traductor, intenta restringirlas al máximo siguiendo un criterio subjetivo sobre su necesidad. En segundo lugar, los lectores prefieren que el texto fluya y aceptan únicamente las notas que son imprescindibles para comprender el texto original. En tercer lugar, las notas metalingüísticas se revelarán como la tipología más necesaria, ya que las referencias de este tipo son quizás las más difíciles de entender sin una nota aclaratoria.

Además de esta introducción, en la que se ha revisado el concepto de paratexto y se han presentado los trabajos más destacados sobre las notas dentro de la traductología, este artículo se divide en tres apartados. En primer lugar, se detalla y justifica la metodología empleada en el estudio. En segundo lugar, se exponen los principales resultados de la investigación cualitativa (editor y traductores) y cuantitativa (lectores) y, finalmente, se presentan las conclusiones, incluyendo las limitaciones del trabajo y posibles futuras líneas de investigación.

\section{Metodología}

En el presente estudio — diseñado a partir de un modelo de investigación mixto—, se optó por dos herramientas clásicas: por un lado, la entrevista al editor de las dos traducciones y, por el otro, un cuestionario a una muestra de sus lectores. Además, se aprovechó el hecho de que las autoras de este artículo fueron también las traductoras de la obra estudiada para realizar una autoetnografía, es decir, incorporar información de primera mano, y así poder triangular los datos.

En cuanto a la entrevista, justificamos su uso acogiéndonos a algunas de las ventajas que listan Fàbregues y otros (2016). En primer lugar, permite obtener información menos superficial, más rica y contextualizada; en segundo lugar, su planteamiento permite aclarar preguntas y respuestas; $y$, finalmente, resulta un contrapunto eficaz a otros datos obtenidos a partir de métodos cuantitativos. En nuestro estudio se diseñó una entrevista semiestructurada para, precisamente, dar cabida a otros aspectos que pudieran surgir y que también pudieran resultar de interés_. Si bien la jefa editorial y, a la vez, la cara más visible de :Rata_cuando se publicó el libro era Iolanda Batallé, la entrevista se realizó al Sr. David Sánchez, editor de 
mesa de :Rata_involucrado, junto con Helena Pons, en la edición de las dos traducciones objeto de estudio.

Cabe recordar que, en el caso de las entrevistas, y tal y como afirman Taylor y Bogdan (1987), el número de entrevistados carece relativamente de importancia, pues lo importante es el potencial de cada caso. El objetivo de realizar la entrevista residía en el conocer de primera mano su postura y práctica editorial en relación con las notas, puesto que los encargos de traducción suelen ir acompañados de la consigna de evitarlas siempre que sea posible y no sabíamos qué criterios habían subyacido en el caso que nos ocupa (Rovira-Esteva, 2007: 17; Casas-Tost y RoviraEsteva, 2008: 221). De hecho, la decisión de incluir más o menos notas depende más del editor - que es quien determina el método traductor y quien, a fin de cuentas, tiene la última palabra- que del traductor, por lo que recoger su voz de alguna manera resultaba imprescindible.

Por otra parte, con el fin de conocer la opinión de los lectores de la obra sobre las notas en general y sobre la necesidad de las notas en la obra analizada, en particular, se diseñó un cuestionario en Google Forms, del que se hizo una versión tanto en español como en catalán para que pudiera ser distribuido entre los lectores de Diarios del Sáhara en el idioma en el que habían leído la obra. Cabe decir que se optó por el cuestionario en línea por sus múltiples e innegables ventajas respecto a las herramientas tradicionales. Varela y otros (2016) consideran que los cuestionarios en línea son una gran alternativa ya que, por ejemplo, garantizan más anonimidad y pueden evitar respuestas incompletas, aunque también cabe reconocer que tampoco están exentos de problemas, pues, por ejemplo, es más difícil controlar si la identidad de los informantes se corresponde con el perfil buscado.

Antes de confeccionar el cuestionario, se analizaron las 26 notas incluidas en las dos traducciones y se clasificaron de acuerdo con la taxonomía de Marrero-Pulido (2001: 80), que distingue los siguientes siete tipos de referencias: geográficas, históricas, culturales, a personajes, intertextuales, intratextuales y metalingüísticas. La traducción de Diarios del Sáhara solo tiene ejemplos de referencias intertextuales, metalingüísticas, de personajes y culturales.

Además de un apartado inicial dedicado a los datos demográficos de los informantes, los cuestionarios constaban de dos secciones. En la primera se les pedía su opinión general acerca de las notas a pie de página a partir de once afirmaciones. Nos interesaba esta información previa, ya que sin duda podía influir sobre el segundo bloque de respuestas y facilitarnos su análisis. Para evitar un cuestionario demasiado largo, que puede convertirse en un obstáculo para la investigación (Varela y otros, 2016), en la segunda parte se les presentó una selección de diez notas, pidiéndoles que indicaran cuán necesaria consideraban cada una de ellas Las

$1 \quad$ La selección final de notas incluidas en el cuestionario se puede consultar en los anexos (1 para el español y 2 para el catalán) de este artículo. 
notas se escogieron proporcionalmente a su peso específico en la traducción y se presentaron de forma intercalada (una de personajes, dos metalingüísticas, tres culturales y cuatro intertextuales).

En ambos bloques los encuestados disponían de una escala de Likert con cinco opciones que iban desde "Totalmente en desacuerdo" (0) hasta "Totalmente de acuerdo" (5), además de la opción de no respuesta.

Los cuestionarios, una vez pilotados, se distribuyeron entre el 29 de marzo y el 6 de octubre de 2018 a través de la red social Twitter, intentando llegar a todos aquellos lectores que hubieran twitteado algún comentario relacionado con el libro o la autora usando la etiqueta “\#Sanmao". Se juzgó necesario buscar informantes que hubieran leído la obra, ya que, aunque no había preguntas de contenido específicas, sí se consideró oportuno que tuvieran una idea global de la misma para poder opinar con cierta perspectiva y conocimiento de causa. Así pues, para poder participar en el estudio se debía ser castellano- o catalanoparlante y haber leído alguna de las dos traducciones. También se pidió colaboración en la difusión de la encuesta a la editorial y a la productora de cine que en esas fechas estaba rodando un documental sobre la autora. Finalmente, se consiguió una muestra de un total de 51 participantes (39 en español y 12 en catalán), dos de los cuales tuvieron que ser descartados por no haber dado su consentimiento informado a participar en el estudio.

Para el análisis cuantitativo se empleó el programa de análisis estadístico SPSS (versión 22). En el caso de las opiniones generales en cuanto a las notas del traductor se ha empleado la prueba de la bondad del ajuste, que permite conocer si una distribución es estadísticamente creíble, mientras que para la comparación sobre cuán necesaria es considerada cada nota se ofrecen medias y medianas, y se ha recurrido a la prueba de rangos con signo de Wilcoxon.

\section{Resultados}

En este apartado se presentan los resultados obtenidos de tres de los agentes más importantes en relación con las notas. Por un lado, la entrevista llevada a cabo con el editor para conocer su política al respecto; por el otro, la experiencia de las traductoras en el caso concreto de la obra que nos ocupa y, finalmente, los cuestionarios respondidos por los lectores, combinando así tanto datos cualitativos como cuantitativos.

\subsection{La política de notas de la editorial como punto de partida}

El Grup Enciclopèdia Catalana es una editorial catalana fundada en 1965. Cuenta con diversos sellos literarios, entre los que se encuentran La Galera, La Galera Young, Catedral, Bridge y :Rata_. Todos ellos tienen políticas diferentes sobre el protagonismo que se le otorga al tra- 
ductor, ya que, por ejemplo, en las obras dirigidas al público infantil y juvenil el traductor no aparece en la cubierta, a diferencia de lo que ocurre en Catedral y:Rata_. Esta última es la que ofrece más visibilidad a los traductores, pues sus libros siempre incluyen una nota biográfica y, a menudo, un prefacio o un epílogo escrito por este.

En cuanto a las notas, el editor observó que la aparición de Internet ha propiciado cambios en este aspecto, puesto que hoy en día al lector le resulta mucho más fácil buscar por sí mismo la información que necesita para comprender el texto y que no está incluida en la obra en forma de nota. En este sentido, le quitó importancia a que no las tuviera. Pese a ello, la editorial considera que las notas metalingüísticas y las intertextuales son las más necesarias porque son las que más cuesta encontrar e, incluso, detectar. Además, el editor manifestó que la presencia de las notas está más justificada en el caso de las traducciones de lenguas cuyas culturas resultan más lejanas al lector meta, como es el caso del chino.

Ninguno de los sellos del grupo tiene una política explícita de notas del traductor: el editor de cada sello decide el umbral de intrusión permitido a través de la inclusión de notas. El editor no suele ofrecer información detallada sobre lo que espera respecto a las notas al hacer el encargo al traductor, si bien sí contextualiza la obra dentro de la colección a la que pertenecerá la traducción para que este se conforme una idea sobre el papel que se le otorga a cada elemento textual. De hecho, el contrato de traducción propuesto por la editorial incluye una cláusula explícita mediante la cual el traductor se compromete a comunicar al editor las notas que pudiera considerar necesarias para obtener su consentimiento.

En definitiva, durante el proceso de traducción el traductor incluye las notas que considera que se precisan y el editor se reserva el derecho a modificar la traducción en su conjunto a partir de la línea editorial y las correcciones técnicas que considere necesarias, aunque siempre hasta cierto punto, pues el traductor también tiene derecho a no reconocer como propia una traducción cuya versión final haya sido alterada en demasía.

Al ser preguntado por los sellos donde es más habitual su presencia, afirmó que no se suelen incluir en la colección para adultos más comercial —Catedral—; de hecho, solo se añaden cuando la información es necesaria para comprender la obra y no se pueda deducir por el contexto. De ser realmente necesario, preferirían incluir dicha información en otro paratexto, como en una introducción. Sin embargo, precisamente porque los paratextos constituyen uno de sus signos de identidad, el caso de :Rata_ es especial. Este sello se ha concebido desde su origen de forma diferente y admite y se nutre de todo tipo de paratextos. Es ilustrativo el hecho de que en el caso de Diarios del Sáhara se incluyeron, además de las notas, un prólogo del escritor Gabi Martínez, una carta de la editora dirigida a la autora, una imagen de un texto con la escritura manuscrita de la escritora, fotos de esta en el Sáhara y un epílogo a cargo de Tai Yufen, una hispanista taiwanesa admiradora de Sanmao, entre otros. 


\subsection{El proceso de creación de las notas por parte de las traductoras}

Las traductoras no recibieron ninguna instrucción explícita sobre la cuestión de las notas, pero en su toma de decisiones eran conscientes de las normas de traducción imperantes en su contexto literario, es decir, que debían evitarlas al máximo. Ambas traducciones se realizaron directamente del chino y, puesto que iban a ser publicadas simultáneamente por el mismo sello editorial, las dos traductoras trabajaron en equipo y consensuaron tanto el número de notas (26 en total) como el contenido de las mismas, decidiendo según su propia experiencia y criterio qué notas eran imprescindibles para la comprensión del texto por parte del lector español medio.

Las traductoras destacan que el trato con la editorial fue de mucho respeto por su trabajo e incluso de un protagonismo no habitual al sentirse siempre una pieza importante del proyecto que se tradujo en una gran visibilidad de su figura con presencia de su nombre en la cubierta, participación en la decisión sobre el orden de los relatos, selección del fragmento de la obra que aparece en cubierta, la inclusión de una biografía, así como un breve texto relatando su relación con la obra traducida. Todo ello las empoderó a la hora de tomar decisiones sobre las notas.

La inclusión de las notas se llevó a cabo calibrando, por un lado, la necesidad que sentiría el lector a la hora de enfrentarse a la obra y, por el otro, intentado simular las mismas condiciones - o conocimientos - que rodean al lector chino medio. Cabe decir que Sanmao no solo es una autora ampliamente afamada en China y Taiwán por sus obras, sino también por su estilo de vida, ya que, en la época, era - y continúa siendo - muy admirada por haberse atrevido a desafiar las normas de una sociedad tradicional y de pautas muy marcadas. Por ejemplo, es bien conocida la anécdota de que Sanmao se negó a regresar a la escuela tras un desafortunado incidente con su profesora de matemáticas, que la humilló públicamente. A raíz de aquel desencuentro, sus progenitores accedieron a tutorizarla ellos mismos desde casa: su padre le enseñaba chino clásico, mientras que la madre le compraba novelas en inglés. A todo ello se le debe sumar su gran pasión por la lectura, que cultivó desde niña. De hecho, la pequeña Sanmao fue capaz de leer Sueño en el Pabellón Rojo, obra maestra de la literatura china, a la edad de cinco años. No es de extrañar, pues, que, bajo el aparente estilo literario sencillo que caracteriza a esta escritora, se escondan un gran número de referencias intertextuales que puedan pasar desapercibidas para los desconocedores de la cultura china. Por ello, las traductoras decidieron no proyectar una imagen de falsa sencillez a lo largo de toda la obra - precisamente para no extender el tópico de que la autora era una ama de casa sin preparación alguna-y decidieron advertir al lector de la presencia de aquellas referencias bajo las que subyacía un profundo conocimiento de la cultura y literatura chinas.

Cabe destacar que la editorial no eliminó ninguna de las notas sugeridas; al contrario, propuso la inclusión de otras tres, de las que finalmente se seleccionaron dos, hecho bastante insólito. En primer lugar, se negoció una nueva nota metalingüística (la número 1 del cues- 
tionario) porque, pese a que las traductoras consideraban que lo importante era percibir el parecido gráfico de los caracteres 人 y 入, sin importar el contenido semántico de estos, la editorial insistió en añadir el significado, petición que tuvo que realizarse a pie de página para no interferir en la lectura. En segundo lugar, la editorial consideró oportuno añadir una explicación referente al relato bíblico de la viuda de las dos monedas. Las traductoras no lo habían estimado necesario por tratarse de una referencia propia de la cultura occidental, pero aceptaron la propuesta. Por último, la nota que se rechazó fue una que pretendía aclarar en qué consistía el complejo de Yocasta, ya que las traductoras concluyeron que por contexto - la estrecha relación entre el marido de la autora y la madre de este- y por estar acompañado del complejo de Edipo en la misma frase, su significado quedaba claro.

Finalmente, otra negociación con la editorial que merece la pena comentar fue la de si mantener o no una explicación sobre qué es el cuscús, que la autora había añadido entre paréntesis en el cuerpo del texto. La correctora propuso dejar dicha explicación como nota e incluso insinuó su eliminación por tratarse de un ingrediente muy conocido en la actualidad. La editorial estuvo de acuerdo con este planteamiento, pero las traductoras opinaron que, pese a tratarse de una decisión editorial, reflejaba un momento histórico en el que el cuscús no gozaba de la misma popularidad en todas partes, por lo que consideraron interesante mantener la susodicha aclaración que, en cualquier caso, formaba parte del texto original, reflexión que la editorial acabó compartiendo.

\subsection{La voz de los lectores}

A continuación, se reporta el perfil demográfico de los encuestados. La edad media de los individuos muestreados es ligeramente superior a los 45 años $(45,2)$. En cuanto al sexo, el $30,6 \%$ son hombres, y el $67,3 \%$, mujeres. Un 2\% declara que su máximo nivel de estudios es la educación primaria; un 10,2\%, la secundaria y el bachillerato; un 6,1\% afirma haber cursado estudios de formación profesional; un 42,9\% declara estar en posesión de un título de grado o licenciatura; un 26,5\% tiene un máster; y un $12,2 \%$ se ha doctorado. El 75,5\% afirma no haber recibido formación en lengua y culturas chinas, mientras que el restante $24,5 \%$ sí tiene algún tipo de conocimiento de ese ámbito. Un 55,1\% de los encuestados ha leído a otros autores chinos o taiwaneses aparte de Sanmao, mientras que el 44,9\% restante declara no haber leído ninguna otra obra procedente de esos territorios. Por último, más de la mitad de los informantes $(67,4 \%)$ lee literatura extranjera frecuentemente o muy frecuentemente, mientras que un $20,4 \%$ lo hace a veces y un $12,2 \%$ raramente.

\subsubsection{Opinión general de los lectores sobre las notas del traductor}

Uno de los objetivos del estudio era conocer la opinión de los lectores acerca de las notas del traductor, ya que tanto editores como traductores toman las decisiones en su nombre cuando, en realidad, apenas existen estudios sobre las notas del traductor desde el punto de vista de su recepción. Así pues, para recoger la opinión de los lectores acerca de las notas, se pro- 
pusieron once afirmaciones valorativas sobre estas. En la tabla I se presentan los resultados del análisis estadístico que permiten discernir si a partir de la muestra pueden extrapolarse conclusiones al conjunto de la población.

\section{TABLA 1}

Resultados sobre la opinión de los lectores acerca de las notas del traductor

\begin{tabular}{|c|c|c|c|c|}
\hline \multirow[b]{2}{*}{ AFIRMACIONES } & \multicolumn{3}{|c|}{$\%$ RESPUESTAS } & \multirow{2}{*}{$\begin{array}{l}\text { RESULTADO } \\
\text { DE LA } \\
\text { PRUEBA DE } \\
\text { BONDAD } \\
\text { DEL AJUSTE }\end{array}$} \\
\hline & $\begin{array}{l}\text { DE ACUERDO } \\
\text { / MUY DE } \\
\text { ACUERDO }\end{array}$ & $\begin{array}{l}\text { EN } \\
\text { DESACUERDO } \\
\text { / MUY EN } \\
\text { DESACUERDO }\end{array}$ & $\begin{array}{l}\text { DEPENDE } \\
\text { DE LA NOTA }\end{array}$ & \\
\hline $\begin{array}{l}\text { 1- Las agradezco porque me ayudan a en- } \\
\text { tender más la obra y su contexto y a dis- } \\
\text { frutarla más }\end{array}$ & 81,6 & 10,2 & 8,2 & $\begin{array}{c}\chi^{2}(2, N=49) \\
=51,469 \\
p<0\end{array}$ \\
\hline $\begin{array}{l}\text { 2- Me parece que aportan información vi- } \\
\text { tal para la comprensión del texto }\end{array}$ & 63,3 & 10,2 & 26,5 & $\begin{array}{c}X^{2}(2, N=49) \\
=21,714 \\
p<0\end{array}$ \\
\hline $\begin{array}{l}\text { 3- Me parecen interesantes para conocer } \\
\text { la cultura del país, que también es uno de } \\
\text { mis objetivos al leer narrativa extranjera }\end{array}$ & 83,7 & 10,2 & 6,1 & $\begin{array}{c}X^{2}(2, N=49) \\
=56,000 \\
p<0\end{array}$ \\
\hline $\begin{array}{l}\text { 4- Me gustan si no interrumpen en exceso } \\
\text { la lectura }\end{array}$ & 71,5 & 16,3 & 12,2 & $\begin{array}{c}X^{2}(2, N=49) \\
=32,122 \\
p<0\end{array}$ \\
\hline $\begin{array}{l}\text { 5- Me parecen bien, siempre y cuando } \\
\text { sean imprescindibles para la compren- } \\
\text { sión del texto }\end{array}$ & 55,1 & 22,4 & 22,4 & $\begin{array}{l}\chi^{2}(2, N=49) \\
=0,510 \\
p=0,475\end{array}$ \\
\hline $\begin{array}{l}\text { 6- Me parecen bien, siempre y cuando } \\
\text { tengan una extensión razonable y sean } \\
\text { accesibles para el gran público }\end{array}$ & 65,3 & 20,4 & 14,3 & $\begin{array}{c}\chi^{2}(2, N=49) \\
=22,816 \\
p<0\end{array}$ \\
\hline $\begin{array}{l}\text { 7- Me parece que aportan información } \\
\text { complementaria prescindible, aunque } \\
\text { prefiero que estén ahí }\end{array}$ & 24,5 & 40,8 & 34,7 & $\begin{array}{l}X^{2}(2, N=49) \\
=2,000 \\
p=0,368\end{array}$ \\
\hline $\begin{array}{l}\text { 8- Me parecen más o menos necesarias, } \\
\text { en función de la cultural original. Cuanto } \\
\text { más alejadas están de la mía, más nece- } \\
\text { sarias me parecen }\end{array}$ & 65,3 & 16,4 & 14,3 & $\begin{array}{c}\chi^{2}(2, N=47) \\
=25,574 \\
p<0\end{array}$ \\
\hline $\begin{array}{l}\text { 9-Son prescindibles y, como tales, me son } \\
\text { indiferentes }\end{array}$ & $2 \%$ & 89,8 & 8,2 & $\begin{array}{c}\chi^{2}(2, N=49) \\
=70,571 \\
p<0\end{array}$ \\
\hline $\begin{array}{l}\text { 10- Me molestan o distraen a la hora de } \\
\text { leer }\end{array}$ & 0 & 85,7 & 14,3 & $\begin{array}{c}\chi^{2}(1, N=49) \\
=25,000 \\
p<0\end{array}$ \\
\hline $\begin{array}{l}\text { 11- No suelo leerlas porque me resultan } \\
\text { aburridas }\end{array}$ & 2 & 79,6 & 18,4 & $\begin{array}{c}\chi^{2}(1, N=49) \\
=49,143 \\
p<0\end{array}$ \\
\hline
\end{tabular}


Del resultado de las pruebas de bondad del ajuste se desprende que en todos los casos los resultados son estadísticamente significativos, salvo en los casos de las afirmaciones núm. 5 ("Me parecen bien, siempre y cuando sean imprescindibles para la comprensión del texto") y 7 (“Me parece que aportan información complementaria prescindible, aunque prefiero que estén ahí”).

Además, se observó que la afirmación con la que los informantes estuvieron más de acuerdo fue la primera ("Las agradezco porque me ayudan a entender más la obra y su contexto y a disfrutarla más”). Esta actitud se confirma, pues la aseveración con la que se mostraron más en desacuerdo fue la novena ("Son prescindibles y, como tales, me son indiferentes"), tal y como muestra la figura 1.

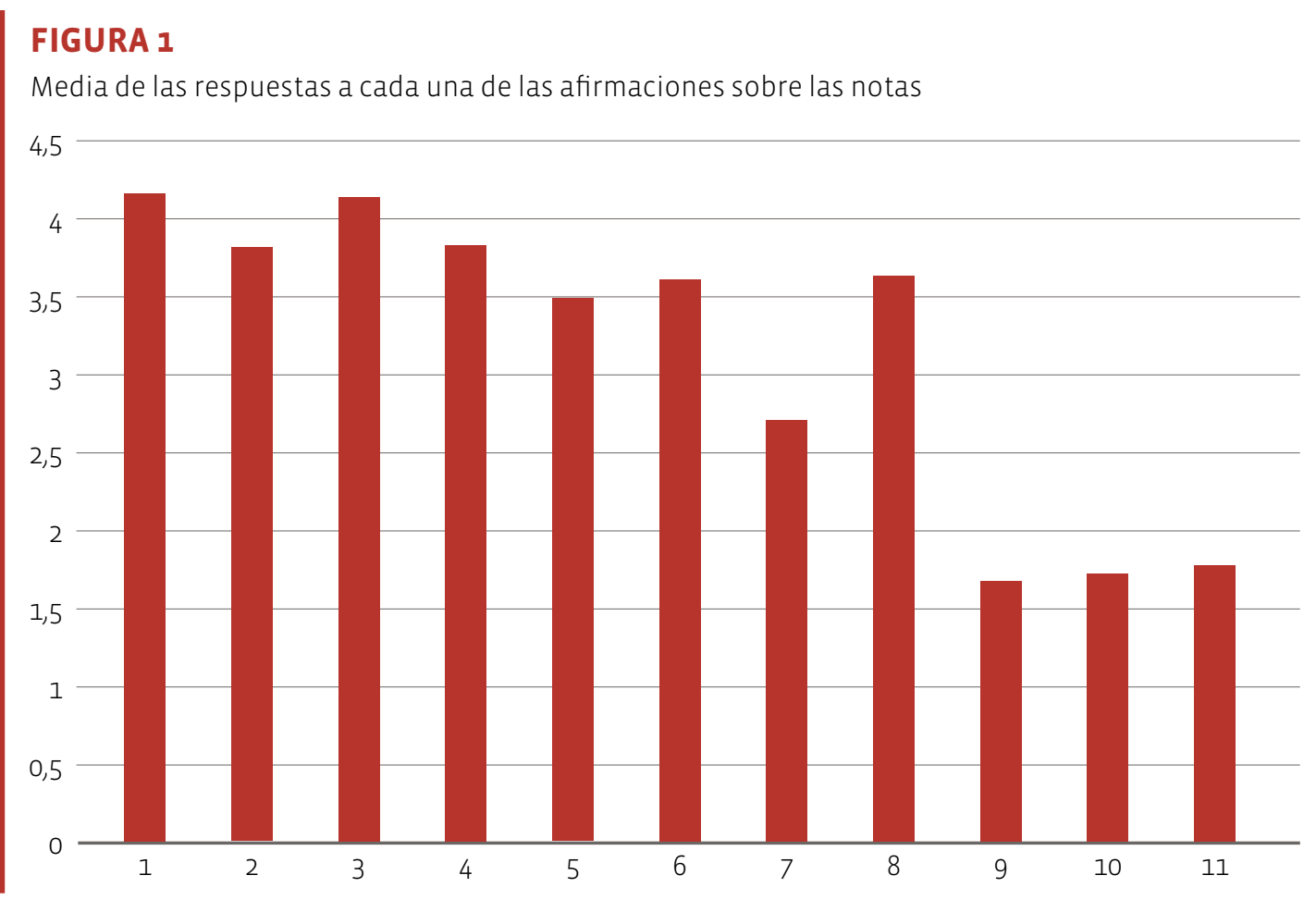

\subsubsection{El caso de las notas de Diarios del Sáhara}

En cuanto a la percepción del grado de necesidad de las notas en Diarios del Sáhara, tal y como indica la figura 2 , vemos que la nota que consiguió la media más alta $(M=4,36$ ) es la décima (metalingüística), pero, si tomamos la mediana como referencia, es decir, el valor más repetido, vemos que la segunda (intertextual), octava (cultural), novena (intertextual) y décima están a la misma altura (Me = 5). De cualquier modo, resulta evidente que, dado que la media más baja es 3,94, se puede afirmar que los informantes consideraron todas las notas propuestas necesarias o muy necesarias. 


\section{FIGURA 2}

Media y mediana de cada una de las notas de la muestra

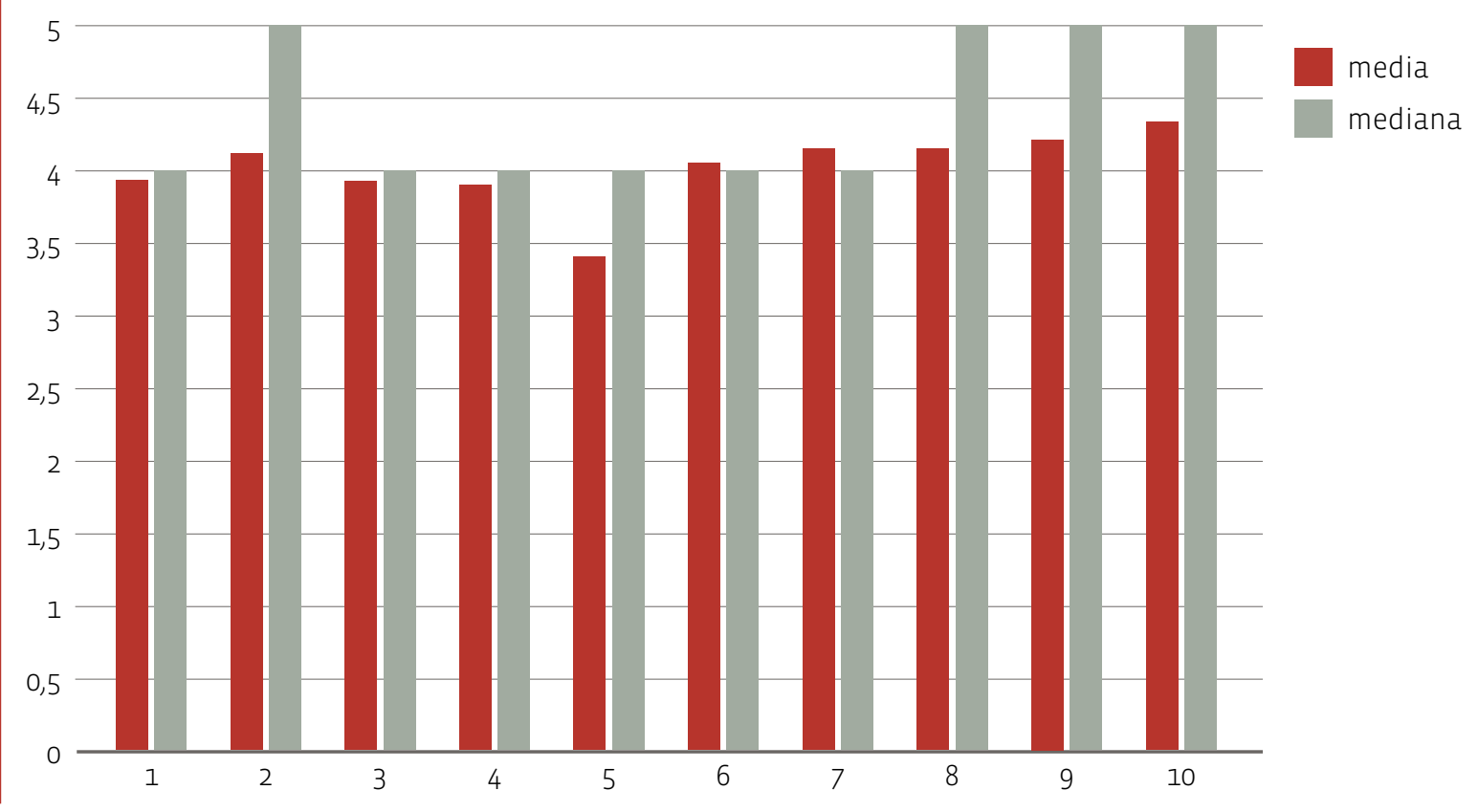

La figura 3 muestra el resultado del análisis de las notas agrupadas por categorías, donde queda patente que los lectores consideran las notas de referencias metalingüísticas e intertextuales las más necesarias de los cuatro tipos incluidos en nuestro análisis.

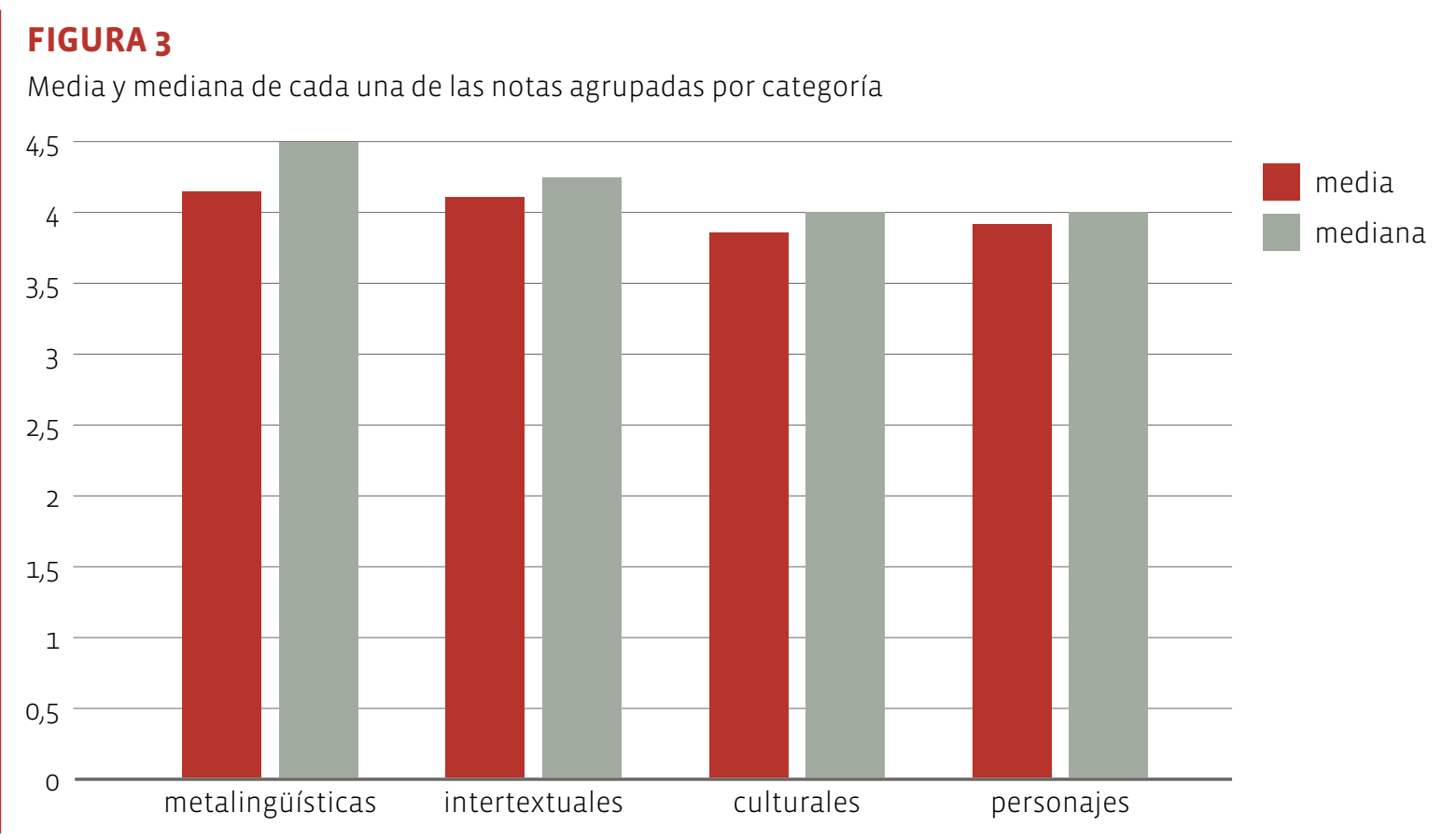


La prueba de rangos de Wilcoxon se llevó a cabo combinando de todas las maneras posibles los cuatro tipos de notas que aparecían en el cuestionario, a saber, notas de referencias metalingüísticas, de personajes, culturales e intertextuales. Tal como se puede observar en la tabla 2, el análisis demuestra que existen diferencias estadísticamente significativas en tres casos: en el de las referencias de personajes e intertextuales, en el que las segundas se imponen a las primeras; en el caso de las referencias metalingüísticas y culturales, en el que el grado de necesidad asociado a las primeras prevalece por encima del de las segundas; y en el caso de las referencias culturales e intertextuales, en el que las segundas se consideran más necesarias que las primeras.

\section{TABLA 2}

Resultados sobre el grado de necesidad de las notas

\begin{tabular}{|c|c|c|}
\hline TIPO NOTA 1 & TIPO NOTA 2 & $\begin{array}{l}\text { RESULTADOS DE LA PRUEBA } \\
\text { DE RANGOS DE WILCOXON }\end{array}$ \\
\hline Ref. personajes & Ref. metalingüísticas & $Z=-1,63, p=0,10$, con 16 empates \\
\hline Ref. personajes & Ref. culturales & $Z=-0,13, p=0,89$, con 10 empates \\
\hline Ref. personajes & Ref. intertextuales & $Z=-2,15, p=0,03$, con 15 empates \\
\hline Ref. metalingüísticas & Ref. culturales & $Z=-2,03, p=0,04$, con 8 empates \\
\hline Ref. metalingüísticas & Ref. intertextuales & $Z=-0,24, p=0,80$, con 14 empates \\
\hline Ref. culturales & Ref. intertextuales & $Z=-2,37, p=0,02$, con 5 empates \\
\hline
\end{tabular}

\section{Conclusiones}

Pese a que las notas son un recurso legítimo para trasladar íntegramente un texto a otra lengua, su inclusión a menudo está cuestionada o no exenta de polémica. Para llevar a cabo este estudio, hemos adoptado un análisis mixto donde, a partir de un estudio de caso, se han triangulado los datos procedentes de los tres agentes involucrados principales: Ios traductores, los editores y los lectores. El enfoque cuantitativo es innovador porque aporta datos objetivos interesantes y abre nuevas vías de estudio centradas en la recepción de las notas.

En cuanto a la actitud general de los lectores respecto a las notas, se ha demostrado estadísticamente que los lectores están más de acuerdo con las afirmaciones positivas acerca de ellas. Solo en dos casos no podemos estar seguros de que los resultados de la muestra no sean fruto del azar. Pese a esta buena disposición de los lectores, los editores parecen ser muy cautos a la hora de incluir notas y en la mayoría de ocasiones las reservan para cuando resultan imprescindibles en pos de la inteligibilidad del texto. Prueba de ello es que el Grup Enciclopèdia Catalana, salvo en el caso del sello :Rata_opta, como criterio general, por prescindir de las no- 
tas siempre que sea posible e intentar introducir esa información en otros paratextos, como, por ejemplo, en un prefacio, o en el mismo cuerpo del texto si no lo distorsiona demasiado.

Otro de los objetivos del estudio era conocer la opinión de los lectores sobre el grado de necesidad de las notas incluidas en Diarios del Sáhara. Nuestro análisis demuestra que el grado de necesidad suele ser, por lo general, considerado alto. Este resultado es coherente con su opinión general sobre las notas. No obstante, las notas metalingüísticas e intertextuales son consideradas las más necesarias, hecho que coincide con la visión de la editorial y las traductoras. De dicho análisis también se desprende que, en comparación, hay algunos tipos de notas que son más necesarios que otros, por lo que estos resultados pueden servir de orientación para editores y traductores a la hora de priorizar notas cuando se ven forzados a reducirlas o limitarlas al máximo.

El presente estudio no está exento de limitaciones. Una de ellas es que la muestra de informantes obtenida no es aleatoria, hecho que, evidentemente, constituye una limitación a la hora de extraer conclusiones y generalizar. No obstante, cabe destacar la imposibilidad o, cuanto menos, la enorme dificultad que entraña conseguir una muestra aleatoria en este caso. Quizás el perfil de lector de una obra china sea más proclive a las notas porque tiene un sesgo al priorizar el valor documental de la obra. En consecuencia, no podemos garantizar, dado el tipo de muestra del que partíamos, que los resultados estén libres de cualquier sesgo. Sería, pues, interesante repetir el análisis seleccionando la muestra de otra manera para poder comparar resultados. Por otro lado, el hecho de que las autoras analicen las notas de una obra traducida por ellas mismas también puede haber sesgado de alguna manera el análisis realizado. Sin embargo, consideramos, por un lado, que las traductoras tenían un papel fundamental en todo este proceso y, por el otro, que era una lástima no aprovechar la información de que disponían de primera mano respecto a su actitud y decisiones en relación con las notas a la hora de triangular todos los datos. Otra limitación de la investigación es que la obra tomada como referencia no presenta ejemplos de todos los tipos de notas inventariadas por Marrero (2001), por lo que no se pueden extraer conclusiones de todas las notas, sino simplemente de las presentes en Diarios del Sáhara. Esta limitación, empero, se puede considerar, a la vez, una nueva oportunidad para dar continuidad a esta investigación y de ampliarla. De hecho, la investigación se enmarca en la primera fase de un proyecto más ambicioso que pretende recoger una muestra representativa de obras chinas traducidas al catalán y al español y de sus notas con el fin de discernir qué tipos de notas son más recurrentes, en qué géneros los traductores recurren más a esta técnica de ampliación o la influencia que pueden tener las lenguas intermedias en el caso de la traducción mediada. También aspira a identificar, mediante un estudio de recepción, qué tipos de notas son los más necesarios para los lectores de manera que traductores y editores puedan escoger con conocimiento de causa las notas que deciden incluir. Por otro lado, resultaría interesante entrevistar a un mayor número de editores para que compartieran sus políticas editoriales en cuanto a este tema y hacer un estudio similar en otro contexto cultural para comparar la influencia de las normas 
de traducción en su sistema literario en las respuestas de los lectores. Finalmente, sería muy recomendable triangular la información preguntando a editores y traductores por su manera de gestionar las notas, tanto cuando han recibido instrucciones de la editorial como cuando lo hacen guiados por su propio instinto.

\section{Bibliografía citada}

CASAs-Tost, Helena, y Sara Rovira-Esteva, 2008: "Claves para interpretar las obras de las 'escritoras guapas' chinas: una aproximación socio-económica y lingüístico-literaria”, Trans. Revista de Traductología 12, 211-230.

El-Madkouri Maataoui, Mohamed, 2001: "Traducción y notas a pie de página” en Anne Barr, María del Rosario Martín Ruano y Jesús Torres del Rey (eds.): Últimas corrientes teóricas en los estudios de traducción y sus aplicaciones, Salamanca: Ediciones Universidad de Salamanca, 158-170.

Fabregues, Sergi, Julio Meneses, David Rodriguez-Gómez y Marie-Hélène Paré, 2016: Técnicas de investigación social y educativa, Barcelona: Universitat Oberta de Catalunya.

FAnCHER, Raymond, 2002: "Editor's review: footnotes pro and con", Journal of the History of the Behavioral Sciences 38 (3), 317-318.

Genette, Gérard, 1997: Paratexts, thresholds of interpretation, Cambridge: Cambridge University Press.

GordeEw, Alona, 2009: Las Glosas Emilianenses y Silenses, s. I.: Grin Verlag.

Henry, Jacqueline, 2000: "De l'érudition à l'èchec: La note du traducteur", Méta 45 (2), 228-240.

Kamal Zaghloul, Ahmed, 2011: “Las notas a pie de página en la traducción del Corán”, Entreculturas 3, 17-36.

López Guix, Juan Gabriel, y Jacqueline Minett WiLkinson, 1997: Manual de traducción inglés-casteIlano: teoría y práctica, Barcelona: Gedisa.

MARÍN-LACARTA, Maialen, 2012: "La recepción de traducciones literarias por su valor documental: El caso de la literatura china moderna y contemporánea en España” en Pilar Martino-Alba y Salud M. Jarilla: Caleidoscopio de traducción literaria, Madrid: Dykinson, 45-56.

Marrero-Pulido, Vicente, 2001: "Información añadida en la traducción literaria: ¿dentro o fuera del texto?” en Isabel Pascua (ed.): La traducción: estrategias profesionales, Las Palmas de Gran Canaria: Universidad de las Palmas de Gran Canaria, 69-88. 
Martin, Alison E., 2008: "Annotation and Authority: Georg Forster's Footnotes to the Nachrichten von Den Pelew-Inseln (1789)", Translation and Literature 15 (2), 177-201.

Molina, Lucía, y Amparo Hurtado-AlBiR, 2002: "Translation Techniques Revisited: A Dynamic and Functionalist Approach”, Méta 47 (4), 498-512.

Newmark, Peter, 1988: A Textbook of Translation, Nueva York: Prentice-Hall International.

Pellatt, Valerie, 2018: "Translation of Chinese Paratext and Paratext of Chinese Translation" en Chris SHel y Zhao-Ming Gao (eds.): The Routledge Handbook of Chinese Translation, London, Nueva York: Routledge Taylor \& Francis Group, 164-85.

Peña, Salvador y M. ${ }^{a}$ José Hernandez, 1994: Traductología, Málaga: Universidad de Málaga, 36-38.

Ribelles, Norma, 2004: "Las notas a pie de página en las versiones al español de las novelas de P. Modiano: ¿La honte du traducteur?", Anales de Filología Francesa 12, 385-393.

Rovira-Esteva, Sara, 2007: "Translating Chinese Pop Fiction”, Perspectives. Studies in Translatology 15 (1), 15-29 [http://dx.doi.org/10.2167/psto02.0].

Sanmao, 2016a: Diarios del Sáhara, traducción de Irene Tor-Carroggio, Barcelona: :Rata_.

Sanmao, 2016b: Diaris del Sàhara, traducción de Sara Rovira-Esteva e Irene Tor-Carroggio, Barcelona: :Rata_.

Sierra, Rocío, 2008: "Notas a pie de página: Reductos de identidad en la sociedad del conocimiento y la globalización" en Assumpta Camps y Lew ZyBatow (eds.): Traducción e interculturalidad: actas de la conferencia internacional Traducción e Intercambio Cultural en la Época de la Globalización, Frankfurt am Main: Peter Lang, 273-284.

TAYLOR, Steven, y Robert Bogdan, 1987: "La entrevista en profundidad” en Introducción a los métodos cualitativos de investigación: la búsqueda de significados, s. I.: Editorial Paidós Básica, 100-132.

Toledano Buendía, Carmen, 2010: “¿Qué hay tras las notas del traductor?” en Rosa Rabadan, Trinidad Guzmán y Marisa Fernández (eds.): Lengua, traducción, recepción: en honor de Julio César Santoyo = Language, Translation, Reception: To Honor Julio César Santoyo, León: Universidad de León, Área de Publicaciones.

Toury, Gideon, 2002: Descriptive Translation Studies - and Beyond, Amsterdam, Philadelphia: John Benjamins Publishing Company. 
Valverde Jara, Xinia, 2017: “Las notas al pie en la traducción”, Letras 61, 181-202 [doi: http:// dx.doi.org/10.15359/rl.1-61.7].

Varela, Carmen, José Ruiz, Ana Andrés, Rubén Roy, Adela Fusté y Carmina Saldaña, 2016: "Advantages and Disadvantages of using the website SurveyMonkey in a real study: Psychopathological profile in people with normal-weight, overweight and obesity in a community sample", E-Methodology, 77-89.

VARnEY, Jennifer, 2007: "Taboo and the translator: a survey of translators' notes in Italian translations of Anglo-American fiction, 1945-2005" en Anthony Pym y Alexander Perekrestenko (eds.): Translation Research Project 1, Tarragona: Intercultural Studies Group, 47-58.

Wu, Pei-Chuan, 2013: En torno a la recepción de la traducción literaria español-chino (Taiwán): un estudio empírico a partir de dos obras de Arturo Pérez-Reverte. Tesis doctoral, Departamento de Traducción e Interpretación, Universidad Autónoma de Barcelona.

\section{Anexos}

\subsection{Anexo 1: Notas del cuestionario en español}

1- Nota núm. 1

«Al ser su mujer no tenía más remedio que soportar su chino. Pobrecito... ¡cuántas veces le expliqué la diferencia entre 人 y 入, y aun así seguía sin diferenciar aquellos sinogramas!»

Nota: Pese a que 人 y 入 son dos sinogramas que se parecen mucho, el primero significa "persona" y el segundo "entrar".

2- Nota núm. 2

«Cuando contestaba las cartas de mis padres, les adjuntaba fotos en las que se me veía despeinada. En el reverso anotaba que aquellos pelos eran como la hierba fragrante, que cuanto más lejos estaba de ellos, más crecía y más se enredaba. En mis fotos también aparecía mi hogar, que se veía muy lúgubre, como una prisión, aunque para mí era el lugar donde hallaba la felicidad: era mi paraíso».

Nota: La autora adapta unos versos de un poema de Li Yu (937-978), gobernante y poeta de la dinastía Tang del Sur durante el Período de las Cinco Dinastías. La obra habla de la tristeza que produce la nostalgia de la separación en los siguientes términos: "el dolor que siento por nuestra separación es como la hierba primaveral, cuanto más lejos estás de mí, más crece”.

3- Nota núm. 3

«[...] y yo por un momento me olvidé de mi tesoro y me puse a su lado a leer tranquilamente por enésima vez $\boldsymbol{A}$ la orilla del agua».

Nota: Novela clásica de la literatura china atribuida a Shi Nai’an (s. XIV). 
4- Nota núm. 4

«Después de colgar los farolillos de papel de mi madre y de pegar en la pared una vívida caligrafía de Lin Huaimin, con caracteres blancos sobre un fondo negro - que correspondían al nombre de la compañía de baile que fundó-, nuestro hogar empezó a tener un toque y una atmósfera indescriptibles».

Nota: Lin Huaimin (1947), bailarín, escritor y coreógrafo taiwanés que fundó la Cloud Dance Company, una famosa compañía de danza contemporánea.

5- Nota núm. 5

«[...] Les invitamos a comer langosta, cangrejo, gambas, almejas y salmón. ¡Y comieron todo lo que quisieron! No estábamos en uno de los mercados nocturnos de Taipéi, sino en Madrid, en una famosísima marisquería de una calle muy animada».

Nota: Los mercados nocturnos son unos espacios comerciales muy típicos de Taiwán, en los que se concentra una gran cantidad de tiendas con oferta de comida rápida a precios populares y están abiertos desde que cae la tarde hasta bien entrada la madrugada.

6- Nota núm. 6

«-Esto... Esto es la primera lluvia de la primavera. Cae en lo alto de las montañas taiwanesas y se congela, hilo a hilo. Los aborígenes la bajan de la montaña cargándola a la espalda y la venden en manojos a cambio de vino de arroz. jEs dificilísima de encontrar!»

Nota: La palabra “aborigen” hace referencia a los habitantes nativos de Taiwán que tienen en común el hecho de no ser étnicamente chinos. Han sido víctimas de los diversos colonizadores de la isla y actualmente la mayoría continúa viviendo en las montañas centrales y en las zonas costeras del este. Pese a los esfuerzos del gobierno en los últimos años para mejorar su estatus socioeconómico, continúan teniendo índices de pobreza y analfabetismo superiores a la media de la población.

7- Nota núm. 7

«- ¡Eres increíble! -exclamaba- Eres como aquel mono, aquel mono que puede transformarse en setenta y dos cosas. ¿Cómo se llamaba, cómo se llamaba...?

Le di un golpecito en la cabeza.

-El Gran e Inigualable Sun Wukong. Y esta vez haz el favor de no olvidarlo».

Nota: Sun Wukong, conocido también como el Rey Mono, es el nombre de un personaje de Xi You Ji («Viaje al Oeste») de Wu Cheng'en (1500-1582). Es una de las grandes novelas de la literatura china que narra la peregrinación del monje Xuanzang (602-664) a la India en busca de unas escrituras budistas. Sun Wukong, uno de los discípulos que lo acompañan, y su protector principal, es un mono dotado de poderes sobrenaturales. Al principio de la obra, el Emperador del Cielo le concede el título de Grande e Inigualable. 
8- Nota núm. 8

«A no ser que mis técnicas de adivinación me hubieran fallado y contra todo pronóstico se tratara de una Guanying (que no estaba claro si era hombre o mujer) o de una Virgen María (que sí había existido, y además era virgen), era imposible que yo le cayera en gracia y que me ganara su cariño. Por desgracia, la madre de mi esposo no pertenecía a ninguna de esas dos clases de mujeres».

Nota: Guanying, una figura importante en el budismo, es como se denomina en chino a la diosa de la misericordia y la compasión. Originalmente un hombre parece que representa a China en forma femenina y maternal por influencia taoísta.

9- Nota núm. 9

«Si hubiera tenido una suegra china, tendría que haber actuado de forma más exagerada: nada más entrar por la puerta tendría que haberme arrodillado y tocado el suelo con la frente una y otra vez. Tampoco era para tanto porque si la suegra era una buena persona te levantaba y no permitía que te quedaras en la intemperie, honrándola durante trescientos días hasta morir congelada».

Nota: Sanmao se refiere a una historia de la dinastía Song (960-1279) que narra cómo Yang Shi y You Zuo, dos estudiosos de los clásicos, fueron a visitar al maestro Cheng Yi para que les resolviera una duda filosófica en la que no se ponían de acuerdo, pero al llegar se lo encontraron meditand y se quedaron esperando pacientemente. Tras una larga espera, el maestro se percató de su presencia y los aceptó como discípulos conmovido por su actitud de respeto.

10- Nota núm. 10

«[...] No paraba de llamarme por el nombre que mis padres y mi hermana mayor utilizaban cuando yo era pequeña.

\section{—iMeimei! ¡Meimei!〉>}

Nota: En chino significa "hermana pequeña”.

\subsection{Anexo 2: Notas del cuestionario en catalán}

1- Nota núm. 1

«Com que m’hi havia casat, no em va quedar més remei que adaptar-me al seu xinès. Pobre home, li vaig explicar mil cops la diferència entre $人$ i $\boldsymbol{\lambda}$, però era incapaç de diferenciar aquests dos sinogrames!»)

Nota: Malgrat que 人 y 入 són dos sinogrames que s’assemblen gràficament, el primer significa "persona" i el segon "entrar". 
2- Nota núm. 2

«Quan responia les cartes als meus pares, els adjuntava fotos en què se'm veia despentinada. Al revers hi anotava que aquells cabells eren com l'herba fragant, que com més Iluny es trobava d'ells més creixia i més se m'enredava. A les fotos també sortia casa nostra, que es veia llòbrega i trista com una presó, tot i que jo em sentia tan afortunada com si estigués al paradís».

Nota: L'autora adapta uns versos d'un poema de Li Yu (937-978), governant i poeta de la dinastia Tang del Sud durant el Període de les Cinc Dinasties (907-960). L'obra parla de la tristesa que produeix la nostàlgia de la separació en els termes següents: "Li hen qiaru chuncao, geng xing geng yuan hai sheng", que literalment significa 'el dolor que sento per la nostra separació és com l’herba primaveral, com més Iluny ets més creix'.

3- Nota núm. 3

«Per un moment em vaig oblidar del meu tresor i era al seu costat llegint tranquil.lament i, per enèsima vegada, $\boldsymbol{A}$ la vora de l'aigua».

Nota: Novel.la clàssica de la literatura xinesa, atribuïda a Shi Nai’an (s. XIV).

4- Nota núm. 4

«A casa nostra es va començar a respirar un ambient difícil de descriure quan vaig haver penjat els fanalets de paper que m’havia enviat ma mare i a la paret es podia admirar la cal.ligrafia en tinta blanca sobre fons negre d'en Lin Huaimin, on es podia llegir "Companyia de dansa La porta dels núvols" i els caràcters de la qual semblaven dracs volant i fènix descansant».

Nota: Lin Huaimin (1947), és un ballarí, escriptor i coreògraf taiwanès que va fundar la Cloud Gate Dance Company, una famosa companyia de dansa contemporània.

5- Nota núm. 5

«Els vam convidar a menjar Ilagosta, cranc, gambes, cloïsses i salmó i van afartar-se com lladres. No ens trobàvem en un dels famosos mercats nocturns de Taipei, sinó en una marisqueria de renom d'un cèntric carrer madrileny».

Nota: Els mercats nocturns són uns espais comercials molt típics de Taiwan en què s’hi concentren una gran quantitat de tendes i parades amb oferta de menjar ràpid a preus populars i estan oberts des que cau la tarda fins ben entrada la matinada.

6- Nota núm. 6

«Això és la primera pluja de primavera. Cau al cim de les muntanyès taiwaneses i es congela, fil a fil. Els aborígens la baixen carregant-la a l'esquena i la venen a manats a canvi de vi d'arròs. No és gens fàcil d’aconseguir!»»

Nota: La paraula 'aborígens' fa referència als habitants nadius de Taiwan que tenen en comú el fet de no ser ètnicament xinesos. Han estat víctimes dels diversos colonitzadors 
de l'illa i actualment la majoria continua vivint a les muntanyès centrals i a les zones costeres de l'est. Malgrat els esforços del govern en els últims temps per millorar el seu estatus socioeconòmic, continuen tenint índexs de pobresa i d'analfabetisme superiors a la mitjana de la població.

\section{7- Nota núm. 7}

«-Ets increïble! -va exclamar- Ets com aquell mico... Aquell mico que pot transformar-se de setanta-dues maneres. Com es deia, que no ho recordo?

—EI Gran i Inigualable Sun Wukong. I aquest cop fes el favor de no oblidar-te'n! —li vaig dir donant-li un copet al cap».

Nota: Sun Wukong, conegut també com el Rei Mico, és el nom d'un personatge del Xi You Ji («Viatge l'Oest») de Wu Cheng'en (1500-1582). És una de les grans novel.les de la literatura xinesa que narra la peregrinació del monjo Xuanzang (602-664) a l'índia en cerca d'unes escriptures budistes. En Sun Wukong, un dels deixebles que l'acompanyen i el seu protector principal, és un mico murri que està dotat de poders sobrenaturals. Al principi de l'obra, l’Emperador del Cel li atorga el títol de Gran i Inigualable.

\section{8- Nota núm. 8}

«Tret que les meves tècniques d'endevinació fallessin i contra tot pronòstic es tractés d'una Guanying (que no estava clar si era home o dona) o d'una Verge Maria (que havia existit i, a més, era verge), era impossible que jo rebés el seu favor i la seva gràcia. Malauradament, la meva sogra no s'ajustava a cap d'aquells dos perfils».

Nota: Guanying és com s'anomena en xinès la deessa budista de la misericòrdia i la compassió. En origen era un home i sembla que es representava a la Xina amb aspecte femení i maternal per influència taoista.

9- Nota núm. 9

«Si hagués tingut una sogra xinesa, hauria calgut actuar d'una manera encara més exagerada. Només entrar a casa, m'hauria d'haver agenollat als seus peus per tocar amb el cap a terra diversos cops. Tampoc era tan greu, perquè no era com passar tres-cents dies honrant-la a la intempèrie fins morir congelada i, si la sogra era bona persona, ella mateixa t'ajudava a aixecar-te».

Nota: Sanmao fa referència a una historia corresponent a la dinastia Song (960-1279) que narra com Yang Shi i You Zuo, dos estudiosos dels clàssics, van anar a veure el mestre Cheng Yi perquè els resolgués un dubte filosòfic en què no es posaven d'acord. Quan van arribar a casa seva se'l van trobar meditant. Tot i que era ple hivern i estava tot nevat, es van quedar fora esperant pacientment per no destorbar-lo. Al cap de molta estona, el mestre se'n va adonar i els va acceptar com a deixebles commogut per la seva actitud tan respectuosa. 
10- Nota núm. 10

«En José estava agenollat al costat del Ilit, a punt de plorar de tan preocupat. No parava d'anomenar-me com els meus pares i ma germana gran quan jo era petita.

\section{-Meimei, Meimei!»〉}

Nota: En xinès meimei significa 'germana petita'. 